CERN-EP/2001-048

12 June 2001

\title{
RESPONSE OF A BGO DETECTOR TO PHOTON AND NEUTRON SOURCES: SIMULATIONS AND MEASUREMENTS
}

\author{
H. Vincke, E. Gschwendtner, C.W. Fabjan, T. Otto \\ CERN, Geneva, Switzerland
}

\begin{abstract}
In this paper Monte Carlo simulations (FLUKA) and measurements of the response of a BGO detector are reported. For the measurements three low-energy photon emitters $\left({ }^{60} \mathrm{Co},{ }^{54} \mathrm{Mn},{ }^{137} \mathrm{Cs}\right)$ were used to irradiate the BGO from various distances and angles. The neutron response was measured with an Am-Be neutron source. Simulations of the experimental irradiations were carried out. Our study can also be considered as a benchmark for FLUKA in terms of its reliability to predict the detector response of a BGO scintillator.
\end{abstract}

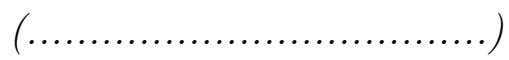


We report on the measurements and simulations of the BGO detector response to calibrated radioactive sources. The energy deposition, which is induced by photons and neutrons in the crystal, is simulated and compared with its measured counterpart.

First we describe the experimental set-up of the low-energy photon measurements including the BGO scintillator. We explain the procedure to obtain simulation results, which can be compared to the measurement. The subsequent part of the paper presents the results and the comparison between simulation and measurement.

Next we report the measurements and simulations of the neutron response. These measurements were carried out with an Am-Be source which emits, together with photons of energy 4.439 MeV, a continuous neutron spectrum. Again, the measurements and simulations are described and an analysis of the comparison presented.

\section{MEASUREMENTS AND SIMULATIONS OF THE BGO RESPONSE TO PHOTONS}

\subsection{Experimental set-up}

The photon response of the BGO crystal was studied with three different radioactive sources. Figure 1 shows the geometry of the experimental set-up and a cross-section through the BGO detector as used in the simulation. For all measurements, three different angles between the symmetry axis of the BGO detector and the position of the source were chosen. The angles, measured from the outer mid-point of the cylindrical crystal cover (see arrows in Fig. 1), were $0^{\circ}, 45^{\circ}$ and $90^{\circ}$. Lack of time prevented a measurement with ${ }^{137} \mathrm{Cs}$ at $90^{\circ}$. The detector was mounted above a table, made of aluminium and polyethylene. The walls surrounding the set-up and the support on which the BGO detector was mounted were neglected in the simulations, whereas the table was included. The chemical formula of the BGO crystal is $\mathrm{Bi}_{4} \mathrm{Ge}_{3} \mathrm{O}_{12}$ and its density can be quoted as $7.13 \mathrm{~g} / \mathrm{cm}^{3}$ [3]. The BGO crystal used was a cylinder with both a height and a diameter of $3.8 \mathrm{~cm}$. The energies and activities of the sources and their distances to the outer mid-point of the cylindrical crystal cover are listed in Table 1. All isotopes used emit also electrons, which are however absorbed in the aluminum encapsulation of the BGO. Details about the measurement procedures can be found in Ref. [2].

Table 1: Radioactive sources used for the photon response measurements (on 7 Dec. 1998).

\begin{tabular}{|c|c|c|c|}
\hline Source & Energy $[\mathrm{keV}]$ & Intensity $[\gamma / \mathrm{s}]$ & Distance \\
\hline${ }^{137} \mathrm{Cs}$ & 662 & $34006 \pm 228$ & $(29.5 \pm 0.1) \mathrm{cm}$ \\
${ }^{54} \mathrm{Mn}$ & 835 & $2294 \pm 15$ & $(10.0 \pm 0.1) \mathrm{cm}$ \\
${ }^{60} \mathrm{Co}$ & $1173.2: 1332.5=1: 1$ & $2 \times(29160 \pm 195)$ & $(29.5 \pm 0.1) \mathrm{cm}$ \\
\hline
\end{tabular}

\subsection{Simulation procedure of the photon response}

For the simulation we used the FLUKA 97 version [1] with the default 'Precision'. With this default setting all relevant physical effects are considered. The energy cut for electrons, positrons and photons was set to $50 \mathrm{keV}$. Neutrons were tracked to $0.00001 \mathrm{eV}$.

In order to obtain simulation results which can be compared to the BGO detector measurements, the simulation has to be performed in two steps.

1) Simulation of the particle histories in the geometry used: the energy deposition induced by each single photon in the BGO crystal is stored in a histogram, with a bin width 

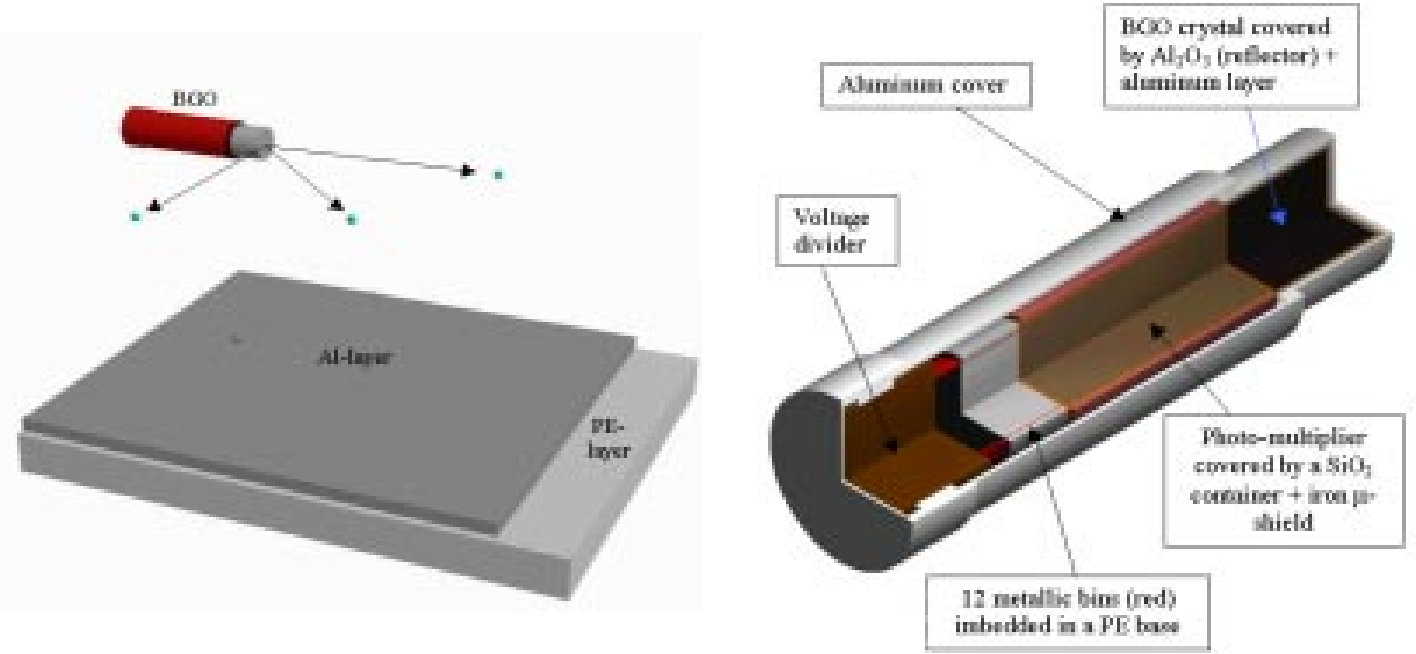

Figure 1: Measurement geometry (left) and cross-section through the BGO scintillator (right). The three small spheres in the left picture indicate the different positions of the source. The connection between the photomultipliers and voltage divider of the BGO detector was implemented in the simulation as a hollow iron cylinder with a mass equivalent to the 12 connection pins.

much smaller than the experimental resolution energy spectrum. Examples of energy deposition spectra produced by this procedure are shown in Fig. 2.

2) In a second step this spectrum was convoluted with the detector-dependent resolution function. The function used has an rms width $\sigma$ of

$$
\sigma(E)=\alpha \cdot \sqrt{E}=1.405 \cdot \sqrt{E(\mathrm{keV})},
$$

where $\alpha$ is a detector-dependent constant, which we measured with a mono-energetic photon source, and $\mathrm{E}$ is the energy deposited in a given bin. The photo-peak energy deposition induced by a ${ }^{137} \mathrm{Cs}$ source $(662 \mathrm{keV})$ including the detector resolution $(36.15 \mathrm{keV})$ at that energy yields a value of $\alpha=1.405$. The convoluted spectra, directly comparable to the measurements, are shown on the right part of Fig. 2. Further details concerning this procedure can be found in Ref. [5].

\subsection{Calculated energy deposition in the BGO detector}

The simulated energy deposition spectra are shown in Fig. 2. For each scenario, the photo-peaks (the photon energy is absorbed completely) and the photo-escape peaks can be found at the theoretically expected energies. A photo-escape peak arises from the escape of an X-ray, which is produced by a secondary reaction of photo-absorption in the crystal. The energy deposited is the total photon energy reduced by the energy of the X-ray. These peaks including their atomic shell dependent structure (K- and L-shell) are reproduced in FLUKA in detail. The ${ }^{60} \mathrm{Co}$ source emits photons with two different energies. Therefore, the above effects occur at two different energies. The next lower energy points in the spectra are the Compton edges. These edges arise from the highest possible Compton scattering energy transfer from a photon to an electron. They emerge at the energies of $477 \mathrm{keV}\left({ }^{137} \mathrm{Cs}\right), 638 \mathrm{keV}\left({ }^{54} \mathrm{Mn}\right), 963 \mathrm{keV}$ and $1119 \mathrm{keV}\left({ }^{60} \mathrm{Co}\right)$.

For a photon energy above $1022 \mathrm{keV}$ the $\left(\mathrm{e}^{+}-\mathrm{e}^{-}\right)$pair production has to be considered. The positron annihilates with an electron of the crystal, resulting in the production 

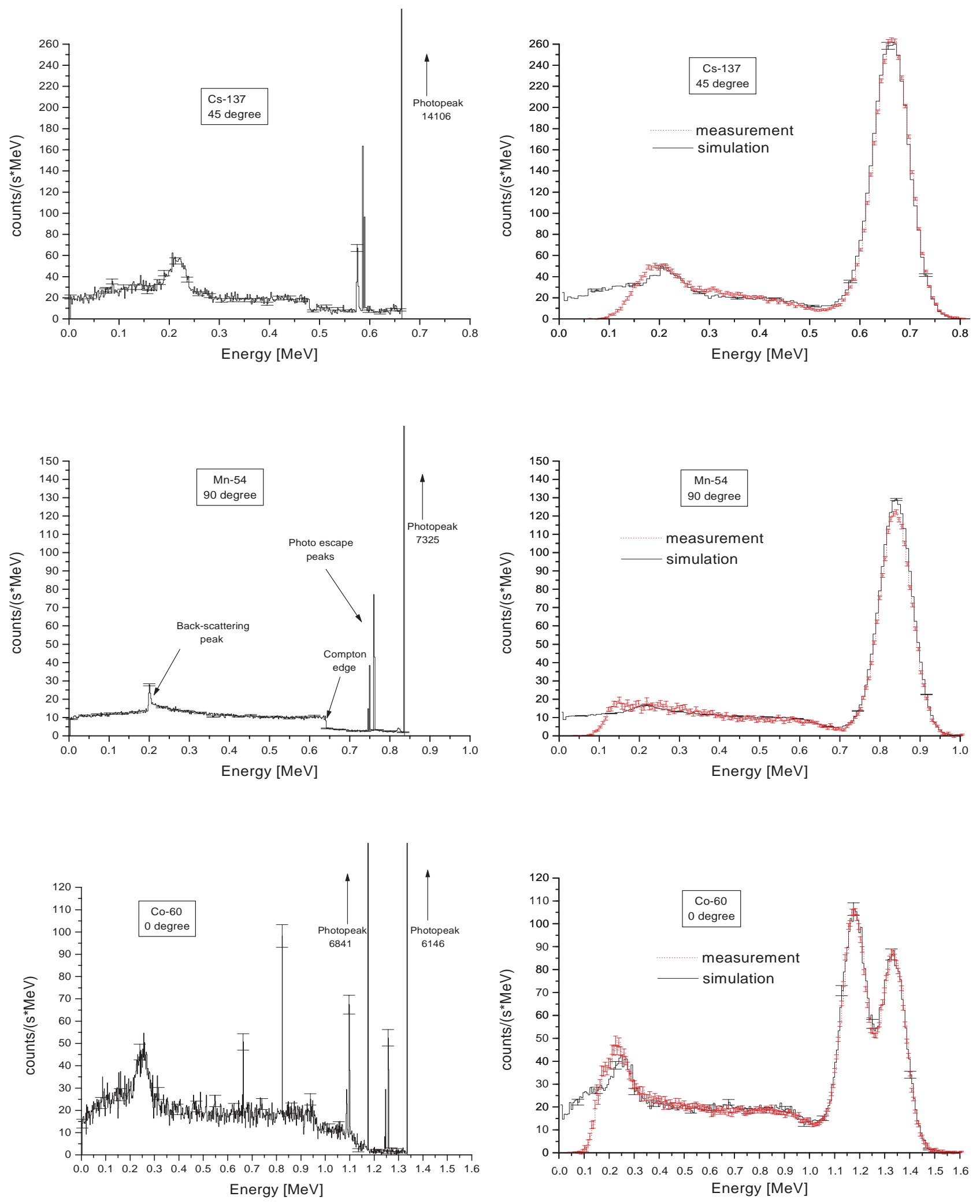

Figure 2: Comparison between measured and simulated spectra. The left side shows the energy deposition spectrum. On the right side the simulation result (solid line) is compared with the measurement (dotted line) after convolution with the energy resolution function. 
of two photons with an energy of $511 \mathrm{keV}$. If one or two of these photons escape the crystal, the total deposited energy is found in the single-escape peak (photo-peak - 511 keV) or the double-escape peak (photo-peak - $1022 \mathrm{keV}$ ), respectively. In the simulation for the ${ }^{60} \mathrm{Co}$ source only the single-escape peaks are visible, because the double-escape peaks are indistinguishable from the energy depositions induced by Compton scatterings.

In all simulations back-scattered photons produce a 'hill' in the range between $170 \mathrm{keV}$ and $300 \mathrm{keV}$. These photons originate mainly from the table located below the BGO detector. In general, the energies of the back-scattered photons reaching the crystal are defined not only by the energy of the primary photon but also by their back-scattering angle. The back-scattering angle range increases with increasing distance between the BGO detector and the source. The reduced distance used for the ${ }^{54} \mathrm{Mn}$ measurement implies a smaller back-scattering angle range than in case of the Co and Cs irradiations. Therefore, the 'back-scattering hill' of the ${ }^{54} \mathrm{Mn}$ exposure extends over a smaller energy interval.

The calculated BGO detector efficiencies concerning energy depositions above $400 \mathrm{keV}$ and the photo-peak efficiencies are listed in Table 2. The efficiency for $E>400 \mathrm{keV}$ is defined as (energydeposits $>400 \mathrm{keV}$ )/(total number of incident particles). The photo-peak efficiency gives the efficiency to absorbe the total incident photon energy.

Table 2: Simulated photo-peak efficiency and efficiency of energy depositions $>400 \mathrm{keV}$.

\begin{tabular}{|c|c|c|c|c|c|c|c|c|c|}
\hline Source & $E$ & Angle & $\begin{array}{c}\text { Efficiency } \\
E>400 \mathrm{keV}\end{array}$ & $\begin{array}{c}\text { Photo-peak } \\
\text { efficiency }\end{array}$ & Source & $E$ & Angle & $\begin{array}{c}\text { Efficiency } \\
E>400 \mathrm{keV}\end{array}$ & $\begin{array}{c}\text { Photo-peak } \\
\text { efficiency }\end{array}$ \\
\cline { 2 - 3 } \cline { 7 - 9 } & $\mathrm{keV}$ & degree & $\%$ & $\%$ & & $\mathrm{keV}$ & degree & $\%$ & $\%$ \\
\hline${ }^{137} \mathrm{Cs}$ & 662 & 0 & $70.8 \pm 0.5$ & $61.8 \pm 0.4$ & ${ }^{60} \mathrm{Co}$ & 1173 & 0 & $62.1 \pm 0.4$ & $40.8 \pm 0.4$ \\
& 662 & 45 & $53.6 \pm 0.3$ & $46.3 \pm 0.3$ & & 1333 & 0 & & $36.7 \pm 0.4$ \\
& 662 & 90 & $64.1 \pm 0.4$ & $55.9 \pm 0.3$ & & 1173 & 45 & $46.2 \pm 0.3$ & $29.8 \pm 0.3$ \\
${ }^{54} \mathrm{Mn}$ & 835 & 0 & $58.1 \pm 0.1$ & $46.2 \pm 0.1$ & & 1333 & 45 & & $26.8 \pm 0.2$ \\
& 835 & 45 & $50.4 \pm 0.1$ & $39.3 \pm 0.1$ & & 1173 & 90 & $55.6 \pm 0.3$ & $36.9 \pm 0.3$ \\
& 835 & 90 & $56.4 \pm 0.1$ & $44.8 \pm 0.1$ & & 1333 & 90 & & $32.5 \pm 0.3$ \\
\hline
\end{tabular}

\subsection{Comparison between simulated and measured results}

Table 3 summarizes the simulated and measured count rates for energy deposition counts with $E>400 \mathrm{keV}$. Above this energy back-scattered photons do not contribute. Measurements and simulations agree, within errors, in all cases.

Table 3: Comparison between simulated and measured count rates $(E>400 \mathrm{keV})$.

\begin{tabular}{|c|c|c|c|c|}
\hline Source & Angle & $\begin{array}{c}\text { Measurement } \\
\text { counts/s }\end{array}$ & $\begin{array}{c}\text { Simulation } \\
\text { counts/s }\end{array}$ & Ratio M/S \\
\hline${ }^{137} \mathrm{Cs}$ & 0 & $24.61 \pm 0.26$ & $24.30 \pm 0.1$ & $(1.01 \pm 0.01)$ \\
& 45 & $27.10 \pm 0.28$ & $27.10 \pm 0.1$ & $(1.00 \pm 0.01)$ \\
\hline${ }^{54} \mathrm{Mn}$ & 0 & $10.52 \pm 0.21$ & $10.84 \pm 0.01$ & $(0.97 \pm 0.02)$ \\
& 45 & $11.53 \pm 0.23$ & $11.83 \pm 0.01$ & $(0.97 \pm 0.02)$ \\
& 90 & $15.43 \pm 0.30$ & $16.20 \pm 0.02$ & $(0.95 \pm 0.02)$ \\
\hline${ }^{60} \mathrm{Co}$ & 0 & $36.46 \pm 0.39$ & $36.50 \pm 0.2$ & $(1.00 \pm 0.01)$ \\
& 45 & $39.52 \pm 0.41$ & $40.00 \pm 0.1$ & $(0.99 \pm 0.01)$ \\
& 90 & $45.56 \pm 0.48$ & $45.20 \pm 0.1$ & $(1.01 \pm 0.01)$ \\
\hline
\end{tabular}

In Fig. 2 three simulated spectra are compared with the measurements. The simulated results were obtained with the procedure explained in Section 2.2. In order to show 
results at representative angles, the comparison for ${ }^{60} \mathrm{Co}$ is shown at $0^{\circ}$, for ${ }^{137} \mathrm{Cs}$ at $45^{\circ}$ and for ${ }^{54} \mathrm{Mn}$ at an angle of $90^{\circ}$. In all three cases good agreeement is obtained. The fact that the real BGO dectector used an energy threshold in order to discriminate the noise explains that the measured spectra do not show counts with energies lower than $50 \mathrm{keV}$. Even at slightly higher energies, the influence of the discriminator threshold is still visible. At low energies the measured back-scattering hill is stronger than the simulated one. This is because the simulations do not consider the influence of the surrounding walls. These walls increase only the number of back-scattered photons with lowest possible energies (back-scattering angle close to $180^{\circ}$ ). At photon energies, which result from $\gamma^{\prime}$ s back scattered from the table, measurement and simulation agree very well again.

\section{BGO DETECTOR RESPONSE TO Am-Be NEUTRON SOURCE}

\subsection{Experimental set-up}

The neutron response of the BGO detector was measured using an Am-Be source. This source emits neutrons with the typical Am-Be neutron spectrum (see Fig. 3), together with photons of an energy of $4.439 \mathrm{MeV}$. The $4 \pi$ neutron rate was measured to be $(2220 \pm 155) \mathrm{s}^{-1}$. The $\gamma$-rate was measured to be $(763 \pm 41)$ photons per second [2].

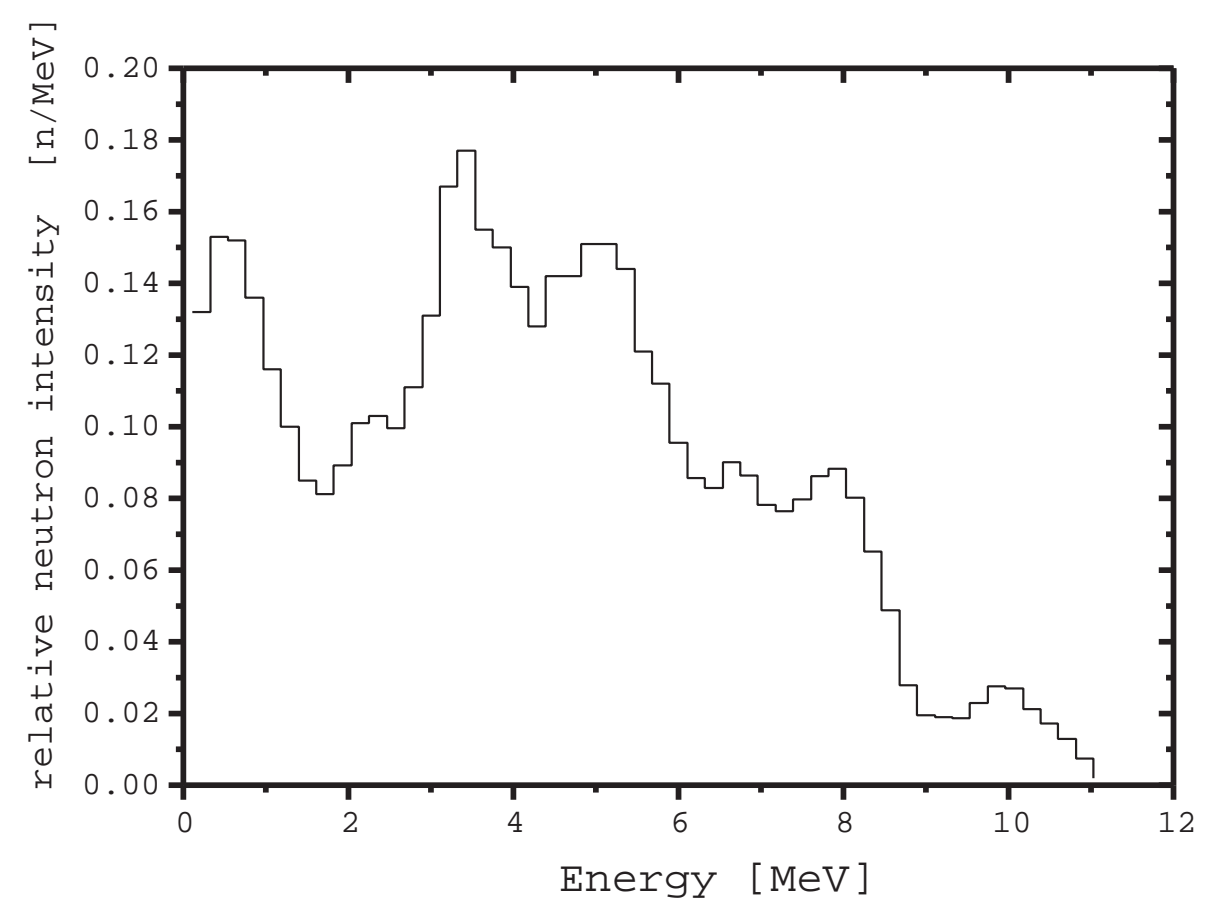

Figure 3: Energy distribution of the emitted Am-Be neutrons.

The cylindrical source, with a height of $10.7 \mathrm{~mm}$ and a diameter of $15.8 \mathrm{~mm}$, was encapsulated in a steel cylinder. The internal structure of this encapsulation and, therefore, the accurate position of the actual source inside its enclosure was, unfortunately, not well known. Based on a sketch of the source, it was assumed that, during the experiment, the center of the Am-Be source was positioned along the BGO detector axis, at a distance of $1.5 \mathrm{~cm}$ from the front face of the aluminium cover. Details about the measurement procedures can be found in Ref. [2]. BGO detector efficiencies for neutrons in the energy range of 4 to $10 \mathrm{MeV}$ have been reported in Ref. [5] 


\subsection{Simulation procedure}

The simulation procedure was performed in three steps. The first two, carried out separately for photons and neutrons, are equivalent to the procedure performed for the $\gamma$-measurements (see Section 2.2). In the third step the simulated total spectrum (sum of photon and neutron counts) was scaled to the measurement. This step was required because of the limited knowledge of the precise position of the Am-Be source inside its Al-cover (see discussion in 3.1). As shown already, FLUKA calculations concerning photon energy depositions in the BGO crystal provide reliable results. We therefore use a scaling factor to account for the source location and adjust the simulated rate to the measured one. Owing to the short distance between source and BGO detector the surroundings of the detector were not taken into account. Details about the simulation procedures can be found in Ref. [4].

\subsection{Simulated energy deposition in the BGO}

The simulated energy deposition spectra induced by Am-Be photons and neutrons are shown in Fig. 4.
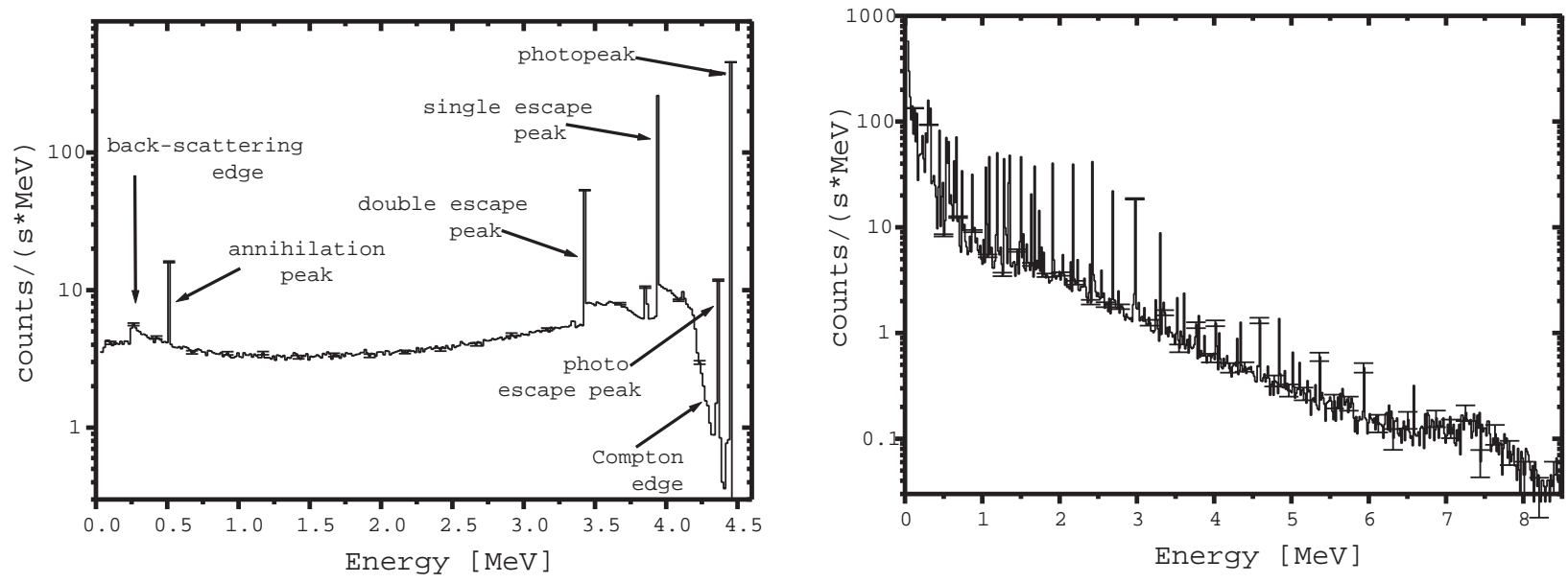

Figure 4: Simulated energy deposition spectrum induced by Am-Be photons (left) and neutrons (right).

In the simulated photon energy deposition spectrum the photo-peak, the photoescape peaks, the single- and double-escape peaks, the annihilation peak and the Compton edge can be found at their nominal energies. An edge at $E=242 \mathrm{keV}$ (back-scattered edge) can also be found in the simulation results. This energy corresponds to the minimum energy of the back-scattered photons. The primary photon crosses the crystal and Compton-scatters behind the crystal. The regions behind the single- and double-escape peaks show a shoulder, explainable by a Compton scattering reaction of one of the $511 \mathrm{keV}$ photons inside the crystal. The small peak at $3845 \mathrm{keV}$ is a combination of single-escape and photo-escape peaks. The total calculated efficiency of the BGO detector response to energy deposits for $E>400 \mathrm{keV}$ is found to be $(37.60 \pm 0.05) \%$. However, the photo-peak efficiency is calculated to be $(8.50 \pm 0.03) \%$. This is because the probability of absorbing the complete photon energy in the BGO detector (photo-peak efficiency) is much smaller for higher primary photon energies.

The neutron energy deposition spectrum exhibits strong peaks in addition to the continuum. This is an artefact of the FLUKA energy-bin structure of low-energy neutron 
interactions. The simulated neutrons, having an energy between 0 and $19.6 \mathrm{MeV}$, are split in 72 energy-dependent groups. Below $19.6 \mathrm{MeV}$, the neutrons do not have a well-defined energy, but rather belong to a specified energy bin. A scattering neutron, belonging to one of these bins, is given a specified probability to be down-scattered into a bin of a lower energy. No matter into which group the neutron is scattered, the computed energy deposition for this simulation step is always the same and is the mean value of all possible energy depositions, induced by scattered neutrons of the relevant energy bin. These sharp peaks in the spectrum correspond exactly to the average value of the possible neutron energy depositions in the BGO detector. At each scattering process, the actual neutron has also a well specified probability to produce secondary neutrons and photons. In case a photon is produced, the energy of this gamma is selected within a given photon energy group structure. The neutron induced photons have, in general, an energy between 0 and $20 \mathrm{MeV}$. This energy range is divided into 22 energy-dependent groups. The actual energy group, chosen for the produced gamma, is selected by given probability distributions, which are assigned to the scattering nuclei and to the energy groups of the scattered neutron. After this group is selected, the photon energy is chosen randomly inside the actual group. The calculated efficiency of the BGO detector for Am-Be neutrons having deposited $E>100 \mathrm{keV}$ is $(20.54 \pm 0.08) \%$. The probability to induce an energy deposition above $400 \mathrm{keV}$ is $(12.01 \pm 0.06) \%$.

\subsection{Comparison between simulated and measured results}

As mentioned in Section 3.2, a normalization factor has to be introduced because of the imperfect knowledge of the source geometry. This factor was obtained by adjusting the integrated counting rates around the simulated and measured Am-Be photo-peaks. Around this peak the counts are dominated by photon-induced effects. In Fig. 5 the convoluted and normalized simulation spectrum is compared with its measured counterpart.

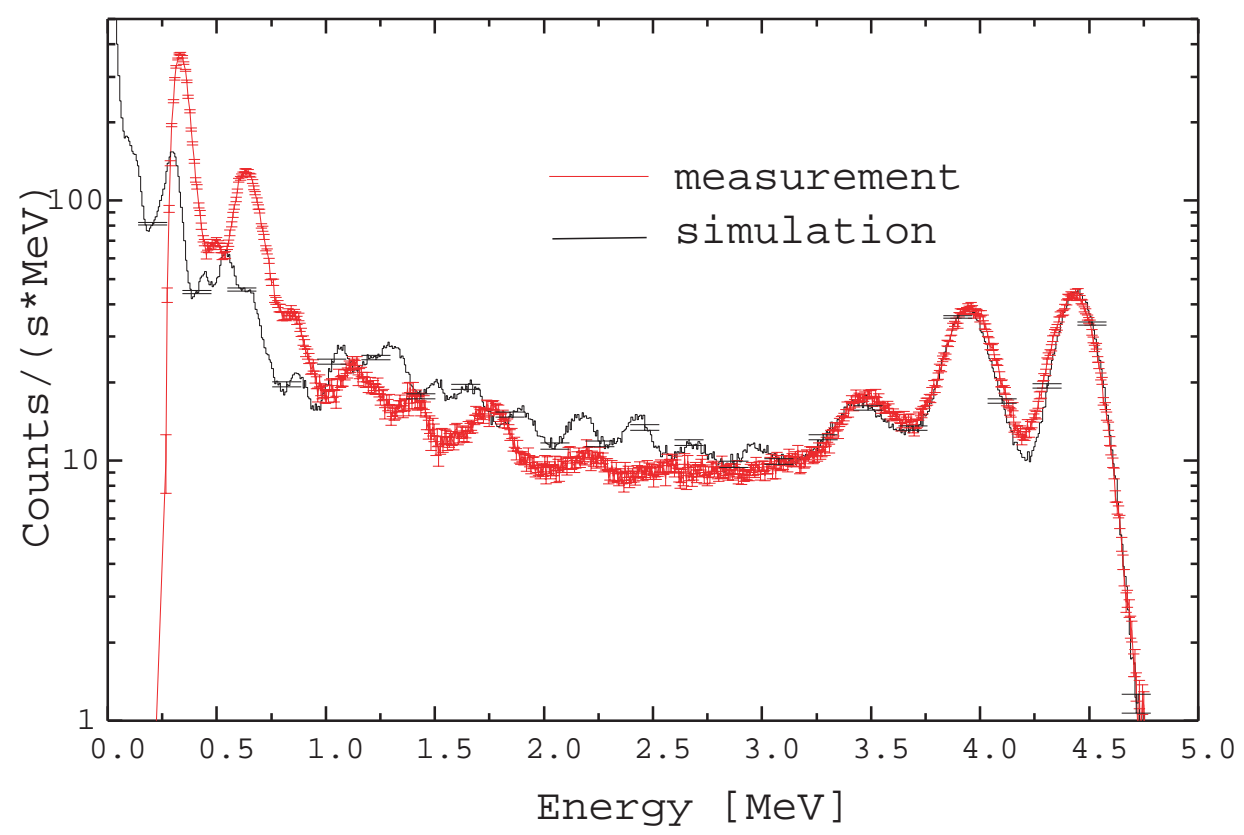

Figure 5: Comparison of measured and simulated BGO detector spectra induced by an Am-Be source. 
The spectrum shows a very good agreement in the range of photon-induced effects. In the lower energy range, which is dominated by neutron-induced energy depositions, discrepancies between the two results can be found. The peaks in the simulated neutron spectrum do not agree with the measured peaks, explained by the simulated energy deposition procedure of neutrons in FLUKA.

Furthermore, the measured peaks, originating mainly from (n,n' $\gamma$ ) processes are simulated only approximately. For example, the measured peak at around $630 \mathrm{keV}$ is induced by inelastic collisions between neutrons and various Ge isotopes. This peak is a combination between (n,n' $\gamma$ ) processes of ${ }^{76} \mathrm{Ge}(563 \mathrm{keV}),{ }^{74} \mathrm{Ge}(596$ and $609 \mathrm{keV}),{ }^{72} \mathrm{Ge}$ $(630 \mathrm{keV})$ and ${ }^{70} \mathrm{Ge}(668 \mathrm{keV})$, which are induced by scattered neutrons. In the simulation only the probability of producing photons in the energy range between $400 \mathrm{keV}$ and $1 \mathrm{MeV}$ is stored. The actual simulated energy of the photon is chosen randomly within this energy range. Therefore, no sharp peaks, originating from neutron-induced photons, can be found in the simulated spectrum.

However, the total rate (integrated counting rate between $10^{-5} \mathrm{eV}$ and $20 \mathrm{MeV}$ ) should be simulated correctly because all necessary cross-sections of $\mathrm{Bi}$, Ge and $\mathrm{O}$ are implemented in FLUKA. Therefore, the probability that a neutron undergoes any kind of reaction inside the crystal is taken correctly into consideration. In Table 4 the simulated and measured counting rates within an energy range of $400 \mathrm{keV}-6.5 \mathrm{MeV}$ are shown. Since the measured and simulated response to low-energy photons agree at the $2 \%$ level (Section 2.4) we can subtract the simulated $\gamma$-rate from the total Am-Be rate to obtain the neutron rate.

Table 4: Simulated and measured BGO detector counting rates (400 keV-6.5 MeV).

\begin{tabular}{|c|c|c|}
\cline { 2 - 3 } \multicolumn{1}{c|}{} & $\begin{array}{c}\text { Simulation } \\
\text { counts/s }\end{array}$ & $\begin{array}{c}\text { Measurement } \\
\text { counts/s }\end{array}$ \\
\hline Total spectrum & $85.1 \pm 3.9$ & $96.5 \pm 0.1$ \\
$\gamma$-spectrum & $44.2 \pm 2.5$ & \\
n-spectrum & $40.9 \pm 3.0$ & $52.3 \pm 2.5$ \\
\hline
\end{tabular}

The main reason for the slight disagreement between measured and simulated neutron rate can be traced to the lower integration threshold $(400 \mathrm{keV})$ on the observed energy deposition.

The measured BGO detector neutron efficiency for an energy deposition above $400 \mathrm{keV}$ is found to be $(15.4 \pm 1.4) \%$. The corresponding simulated value is $(12.01 \pm 0.06) \%$.

\section{CONCLUSION}

We have studied the response of the BGO detector to photons and neutrons.

The first part dealt with the BGO detector response to low-energy photons. Three different radioactive sources, namely ${ }^{137} \mathrm{Cs},{ }^{54} \mathrm{Mn}$ and ${ }^{60} \mathrm{Co}$, were used in order to irradiate the detector. The energy deposition in the BGO crystal was simulated in detail. Physical effects, like pair production, Compton scattering and photo-effect, were reproduced in the simulation with high accuracy. A comparison of the simulated and measured rates yields an agreement mostly better than $2 \%$. The simulated spectra show good agreement with their measured counterparts in all energy regions. Effects, like photo-peaks, Compton edges or single- and double-escape peaks, coincide in all details with the proper measured values. We conclude that we understand the absolute photon response at the $2 \%$ level. 
In the second part, the response of the BGO detector to Am-Be source particles was simulated. The simulation of the energy deposition spectrum induced by Am-Be photons agrees very well with the measurement. The simulation of the energy deposition induced by neutrons can be summarized as follows: The integrated rates between $400 \mathrm{keV}$ and 6.5 MeV agree at a level of better than 30\%. This discrepancy between the measured and simulated rates is mainly due to the high value of the integration threshold (400 keV). The simulated neutron spectra cannot be directly compared to measurement because of the approximations used in the FLUKA simulation procedure. It should be stressed however that the FLUKA simulation procedure evaluates correctly integral neutron interaction effects, such as the total neutron counting rate. Integral neutron efficiencies can therefore be quite reliably obtained with FLUKA. More generally, our studies provide a benchmark of FLUKA for low-energy $\gamma$-interactions and integral low-energy neutron interactions. This is important for a range of simulation studies in natural science, geography and medicine.

\section{Acknowledgement}

We gratefully acknowledge the excellent support provided by G. Roubaud and J. Bourges, and the interest of Prof. Hj. Müller.

\section{References}

[1] A. Fasso et al.: New Developments in FLUKA Modelling Hadronic and EM Interactions, Proc. 3rd Workshop on Simulating Accelerator Radiation Environments, KEK, Tsukuba, Japan 7-9 May 1997, Ed. H. Hirayama, KEK Proceedings 97-5, p. 32-43 (1997).

[2] E. Gschwendtner, Benchmarking the particle background in the LHC experiments, Ph. D. thesis, CERN (2000).

[3] F.G. Knoll: Radiation Detection and Measurement (Third Edition), John Wiley\&Sons, New York, USA (1999).

[4] O. Häusser et al., Nucl. Instrum. Methods 213 (1983) 301.

[5] H. Vincke, Benchmarking of the simulation of the ATLAS hall background, Ph. D. thesis, CERN (2000). 